\title{
CANNABIDIOL VERSUS ALPRAZOLAM AS PREMEDICATION IN TERMS OF POSTOPERATIVE PAIN SCORES AFTER TOTAL KNEE ARTHROPLASTY
}

Doka P., Papaioannou E., Koziakas E., Analyti A., Maniati A., Papadopoulou E., Lampadariou $A$.

Department of Anaesthesiology and Pain Management, "Laikon" General Hospital of Athens

Aim of this study was the evaluation of cannabidiol (CBD) versus commonly prescribed alprazolam as ppremedication, in terms of postoperative pain.

\section{Material and Methods}

In a perspective randomized study 56 scheduled to total knee arthroplasty, under subarachnoid anaesthesia, were divided in two groups, Group A $(n=28)$ patients received CBD 50mg capsule and group B $(n=28)$ alprazolam $0.5-1 \mathrm{mg}$ tablet per os, as premedication on the morning of operation. Both groups'patients received tramadol 50 $100 \mathrm{mg} \times 4$, and parecoxib $40 \mathrm{mg} \times 2 / 24 \mathrm{hr}$ iv. We recorded patients' demographics, operation duration, intra- and postoperative surgical complications, and pain scores postoperatively $1,6,12,24$, 36,48 and 72 hours after end of subarachnoid anaesthesia. Other perioperative complications, such as nausea, vomiting, haemodynamic variations, as well as need for extra analgesics postoperatively were also recorded.

\section{Results}

Demographics and operation duration did not differ significantly between two groups. No surgical complications were recorded. On the first, $6^{\text {th }}, 12^{\text {th }}$ and $24^{\text {th }}$ hours postoperatively, pain scores were significantly lower $(p<0.005)$ in group $A$ $(0.5 \pm 0.08$ vs $2.9 \pm 1.3,1.1 \pm 0.6$ vs $3.4 \pm 1.7$, $1.0 \pm 0.2$ vs $3.6 \pm 1.6,0.7 \pm 0.05$ vs $2.7 \pm 1.4$ in groups $A$ and $B$ respectively on first 24 hours of study), while there was no difference 36, 48 and 72 hours later. Need for extra analgesia was significantly higher $(p<0.005)$ in group $B$ at all times of study (plus $250 \pm 22$ vs $870 \pm 54 \mathrm{mg}$ tramadol iv in groups $A$ and $B$ respectively) .6 group $A$, and 11 group $B$ patients demonstrated nausea $(p<0,005)$, and all of them were haemodynamically stable.

\section{Conclusion}

CBD as premedication seems to provide lower pain scores intra and 24hours postoperatively, compared to commonly prescribed alprazolam. Further study is needed to support this conclusion. 